\title{
PARQUE DE LA DEMOCRACIA Y DE LA JUVENTUD CIUDAD DE RESISTENCIA, CHACO
}

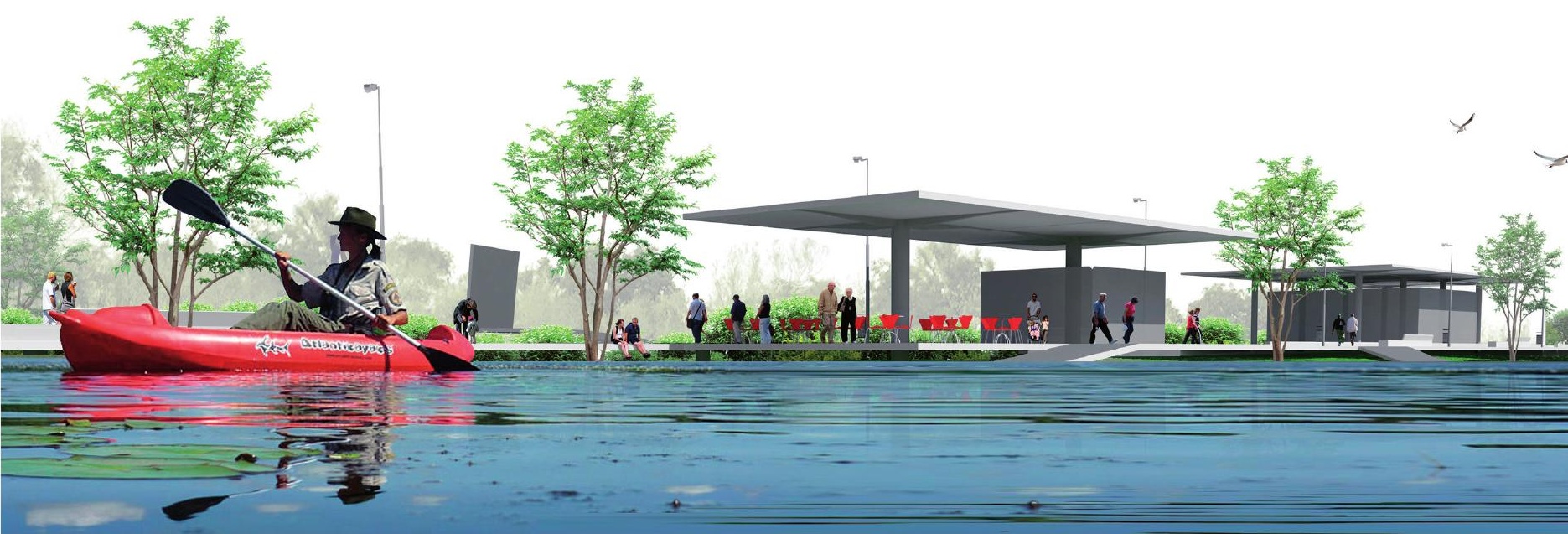

ADRIAN PRIETO PIRAGINE, nace en la ciudad de Resistencia, provincia del Chaco, Argentina. Arquitecto, egresado de la Universidad Nacional del Nordeste. Es socio-fundador y CEO de ARQOM®, joven empresa creativa Argentina con base en la prov. del Chaco, dedicada a innovar, crear y desarrollar de forma sostenible la acción proyectual en distintas áreas del diseño, con reconocimiento a nivel nacional e internacional por su enfoque del diseño. Responsable de la coordinación de los proyectos y el desarrollo de innovaciones. A partir de la interacción entre las distintas disciplinas (diseño de productos, micro-urbanismo, arquitectura, interiorismo y comunicación), logra gran experiencia que le permite moverse con fluidez dentro de los procesos interdisciplinarios de diseño, desarrollo de proyectos y productos.

En forma paralela, es miembro activo de UICH Joven de la Unión Industrial de la Prov. del Chaco y se desempeña como consultor especialista en micro urbanismo, equipamiento y espacio publico, en la Confederación Argentina de la Mediana Empresa (CAME). 


\section{MEMORIA DESCRIPTIVA \\ CARACTERÍSTICAS DEL ÁREA DE IMPLANTACIÓN}

\section{Localización}

El predio destinado a la implantación del parque se localiza en el área norte de la ciudad de Resistencia. Cuenta con una superficie de $207.132,47 \mathrm{~m}^{2}$, de los cuales $66.513,17 \mathrm{~m}^{2}$ se encuentran afectados a laguna. Esta zona se asocia al paisaje característico del valle fluvial del Río Negro, extensos humedales, vulnerables a inundaciones. Las cotas del terreno varían entre 46,50 y 49,50m, encontrándose en un área de riesgo hídrico "restricción Severa" donde se sugieren usos recreativos, siempre que no impidan el natural escurrimiento de las aguas en el sector.

Su entorno urbano se caracteriza por usos residenciales de baja densidad, locales recreativos nocturnos y comercios de gran escala, a lo que se suma la complejidad de la Av. Sarmiento, vía principal de acceso a la ciudad utilizada como eje recreativo-deportivo, de uso frecuente y lugar de encuentro social de la juventud los fines de semana.

\section{Geomorfologías y suelos}

Los suelos de la zona se caracterizan por ser arcillosos, en donde el agua no penetra en el terreno, formando extensos bañados y esteros. El suelo característico de la ciudad es de origen aluvial en general. En algunos sectores, comportándose como activas con fuertes cambios volumétricos por modificación en su contenido de humedad. Esto produce el movimiento de las construcciones livianas con la aparición de fisuras en paredes y pisos. La infraestructura de la ciudad no está exenta de la acción que promueven los suelos. Las redes de distribución de agua, las colectoras de líquidos cloacales y los desagües pluviales se ven alterado por fisuras o microfisuras que producen el lavado de los suelos y posterior hundimiento de veredas y pavimentos.

\section{Paisaje natural}

Los paisajes reconocidos en el área pueden clasificarse como:

Curso del río Negro: monocanalizado, meandros cortados y lagunas subcirculares someras. La vegetación es escasa en el curso principal. Especies de alta tasa de renovación. Frecuentemente carrizales y maciegas controladas por la estacionalidad del flujo y por la salinidad que se da en el período de aguas bajas.

Lagunas: Predominan lagunas semilunares y de 0,50 a 1,50m de profundidad en aguas bajas $(2,00$ a 3,00m en aguas altas), con cubetas de forma de " $v$ " o de "u" y aguas cargadas de materia orgánica. Predomina la vegetación arraigada de hojas flotantes y flotante libre, llegando a cubrir la mitad o todo el cuerpo de agua. Son el hábitat permanente o temporario de numerosas aves acuáticas.

Bañados: Depresiones centrales. Permanecen secos varios meses, aunque pueden tener hasta $2,00 \mathrm{~m}$ de agua sobre el suelo. El agua tiene poca circulación. La vegetación está constituida por plantas de rápido crecimiento y alta productividad. Un solo estrato (1,50 a 2,50m de alto), cobertura densa y cerrada.

Esteros y juncales: Planicies levemente cóncavas, impermeables, en las que el agua queda retenida al menos 8, 10 meses en el año. No tienen forma definida ni límites fijos. Generalmente ocupan bañados bajos, o el anillo lateral de algunas lagunas y arroyos de escurrimiento muy lento. La vegetación son "juncales", formaciones vegetales. Los esteros tienen dominancia de otras especies neófitas.

Bosques en galería: Ubicados en albardones altos que sobresalen 1,00 a 1,50m respecto del resto de la llanura externa al río. La vegetación está conformada por un bosque alto pluriespecífico, con 3 o 4 estratos leñosos en el que coexisten especies propias de la región Chaqueña Oriental con las típicas del ambiente fluvial. 


\section{.... DIÁLOGO ENTRE EL PARQUE Y LA CIUDAD}

\section{... Se habita un territorio de ríos}

Carece de sentido hacer propuestas de buena calidad arquitectónica si están puestas sobre terrenos degradados, sobre todo si la degradación proviene de la misma especie que produce esas propuestas de buena calidad. Esto quiere decir que mientras no se tenga conciencia de que se habita un territorio de ríos y ese territorio de ríos no sea tratado como tal, podemos decir que no avanzamos nada. "Tratemos bien al río, y el resto se nos dará por añadidura"...

Esta propuesta no busca salvar Resistencia, sino recomponer una parte del territorio urbano que se está usando mal, y corregir el uso partiendo de prácticas que permitan conocer el funcionamiento de la naturaleza a fin de modificar nuestro comportamiento colectivo cotidiano. Nuestra propuesta consiste en conectar las partes sueltas del río y hacerlo funcionar como tal.

\section{SITUACIÓN HÍDRICA}

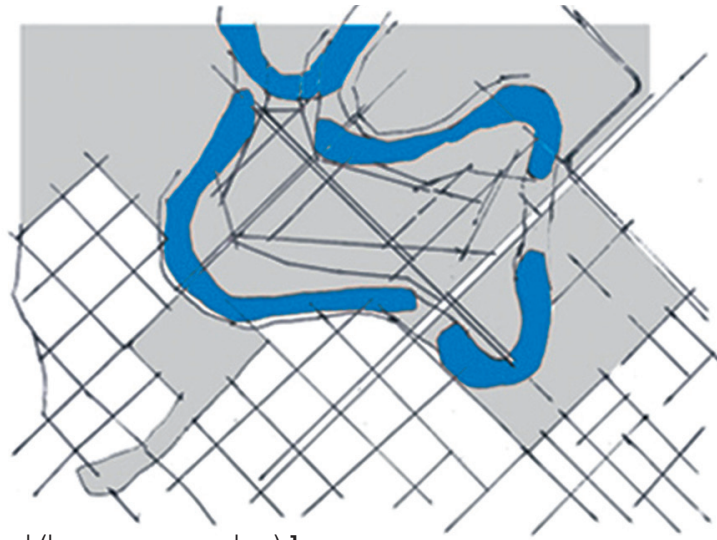

[Actual (lagunas y meandros) ]

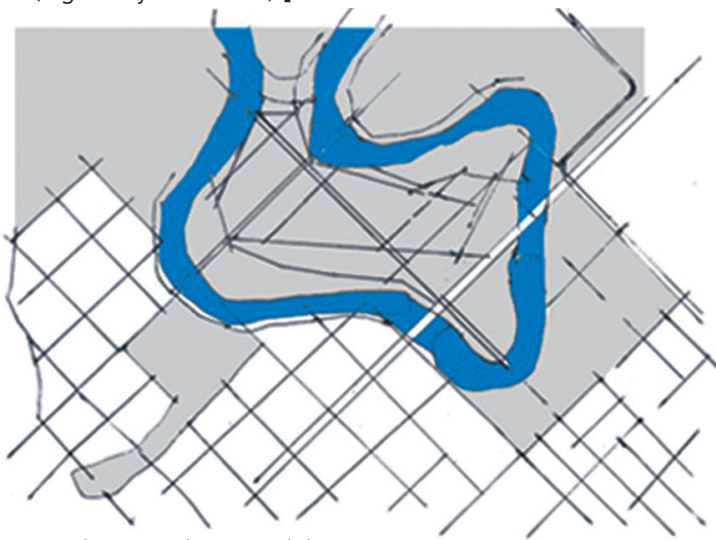

[Propuesta de recanalización del río]

\section{... UN BUEN LOTE SANEADO, EN DOS ESCALAS DE INTERVENCIÓN}

Al hacer funcionar el río y conducir el agua se consolidan los bordes y con ello aumentan la dimensión y la utilidad del lote. Si bien responde a las medidas indicadas para definirlo como parque, queda chico a las necesidades del área urbana considerada en las bases. Por ello creemos necesario aprovechar la sanidad por obtener y proponemos que sea allí donde se construya el edificio de la legislatura, agregando además el factor que es parte de esta propuesta de conectar un buen lote saneado a la trama de la ciudad, para hacerlo accesible y con ello extender la superficie disponible de suelo urbano.

Cumplido esto, se ajustan los reglamentos y se retoma la cultura del río, sin intentar negarlo cuando lo rellenamos, ni cortar su cauce y menos sentirnos "únicos propietarios" cuando lo cruzamos con alambrados.

\section{... DE NUEVO LA IDEA, IDEAS POSIBLES, IDEAS DENTRO DE LA MISMA IDEA}

En la intervención a escala urbana, es necesario recomponer los bordes de circulación de agua - más allá de los límites del terreno-, recortados por la colonización equivocada de un territorio con ríos de llanura. En este sentido, la Av. Sarmiento se comporta como elemento de costura que permite el paso sobre la depresión del perfil, revalorizando su condición de doble vía de conexión: para la ciudad y para la región.

Otra estrategia que emplear para lograr la conexión del sector al sistema vehicular y evitar su aislamiento fue prolongar la calle Liniers, que desemboca en un puente ubicado sobre el brazo de agua. Esto permite por un lado superar la barrera que impone el tráfico vehicular, y por otro, lograr un acceso alternativo al lote.

Hacia el interior del lote se decidió... respetar la fuerza rectora de la trama urbana... y componer espacios soportes cuya estructura sea fácilmente reconocida por los habitantes de la ciudad. 


\section{...LA IDEA SE ESTRUCTURA MEDIANTE VÍAS PERPENDICULARES DE CIRCULACIÓN Y TRES PARQUES DE DIFERENTE CARÁCTER}

Las troncales circulatorias sirven para relacionar las distintas áreas, y al mismo tiempo delimitar los usos previstos en el programa.

El eje NO-SE (se asemeja a la línea recta de la ruta 16 como elemento que "cose" la pcia. en línea recta) atraviesa el predio de borde a borde del meandro con circulación peatonal, se encuentra sobreelevado para permitir que el agua fluya en momentos de crecida, transformándose en un elemento reductor de velocidad en la Av. Sarmiento, dando prioridad al peatón. Este eje arranca en el agua y termina en el agua.

El eje NE-SO da continuidad a la calle Liniers, buscando extender la trama urbana.

Para el recorrido que sigue la traza del meandro se prevé un sistema mixto, con una troncal de circulación vehicular central con sendas peatonales, de skate y bici-senda, separadas de la vehicular por resumideros en forma de cordón cuneta y protegidas de la bajada al río por un entepado plano.

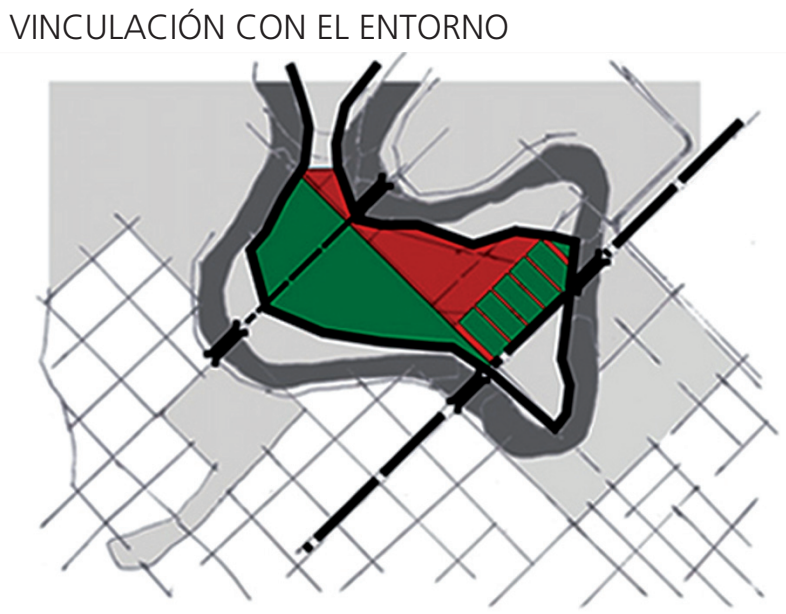

[Circulación peatonales/ vehiculares.]

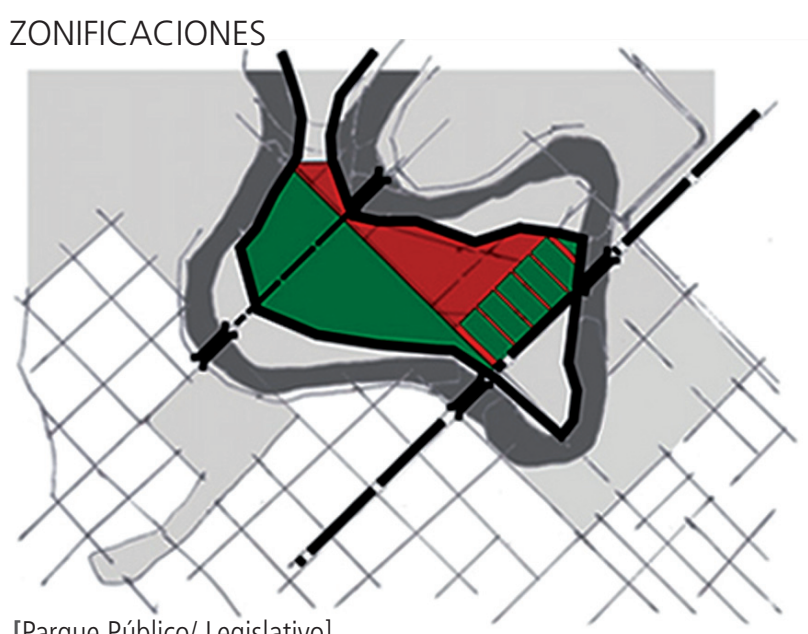

[Parque Público/ Legislativo]

\section{... EN CONVIVENCIA CON}

LA NATURALEZA

La naturaleza es la base para desarrollar la propuesta por medio de una intervención pasiva del espacio (bajo impacto ambiental - sistema palafítico), conservando los árboles existentes y proponiendo la forestación de especies nativas (árboles), en áreas donde estos no existieron por su sombra, con el objetivo de mejorar, restaurar y conservar espacios naturales degradados. 2 . Impulsando la acción ciudadana en defensa del medio ambiente, participando en acciones forestales, sensibilizando a la población, incentivando la participación social y promoviendo la educación ambiental.

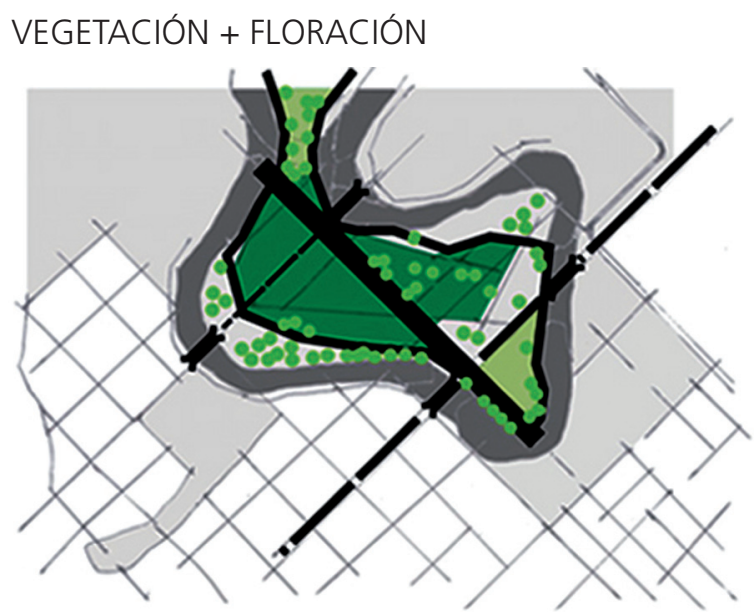

[Existentes (autóctonas).] 


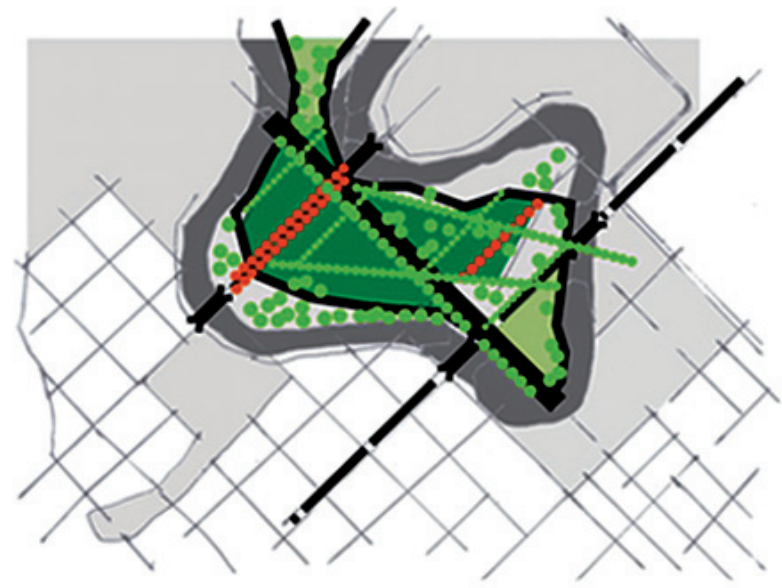

[Inicio verano (especie: chivato)]

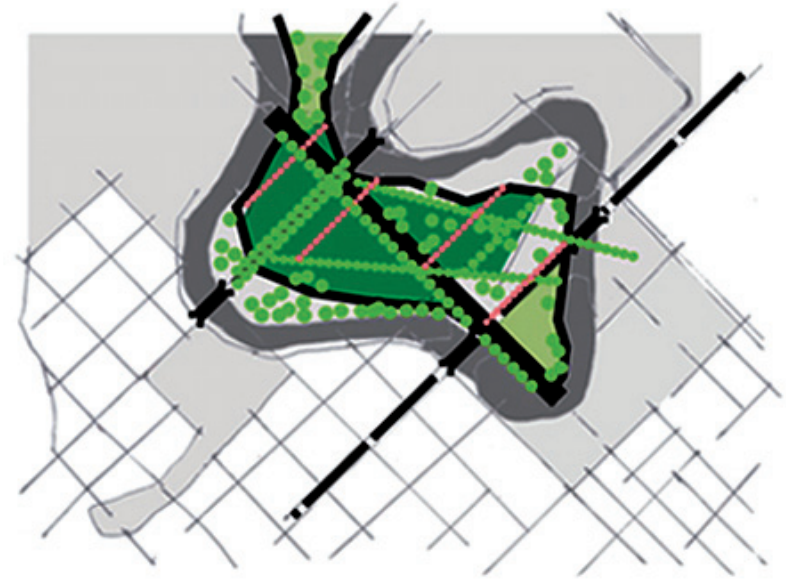

[Inicio primavera (especie: pata de buey)]

\section{... ESPACIOS FLEXIBLES, FUNCIONALES Y UN EDIFICIO HITO PARA LA LEGISLATURA}

Sobre el eje NO-SE, y a modo de acceso desde la Av. Sarmiento, se ubicó el parque cívico-popular, destinado a actividades relacionadas con el ejercicio de los derechos ciudadanos.

La explanada del parque se extiende hacia adentro del lote, donde la propuesta plantea la posibilidad de implantar el edificio de los legisladores, como hito principal de referencia. Se levanta en medio de la naturaleza, enmarcando el acceso a la ciudad, símbolo de la vigencia y fuerza de nuestras instituciones democráticas. El edificio "vuela" al dejar una planta libre y elevarse sobre columnas de hormigón que replican los árboles dispersos en el lote, respetando la esencia de sitio libre,

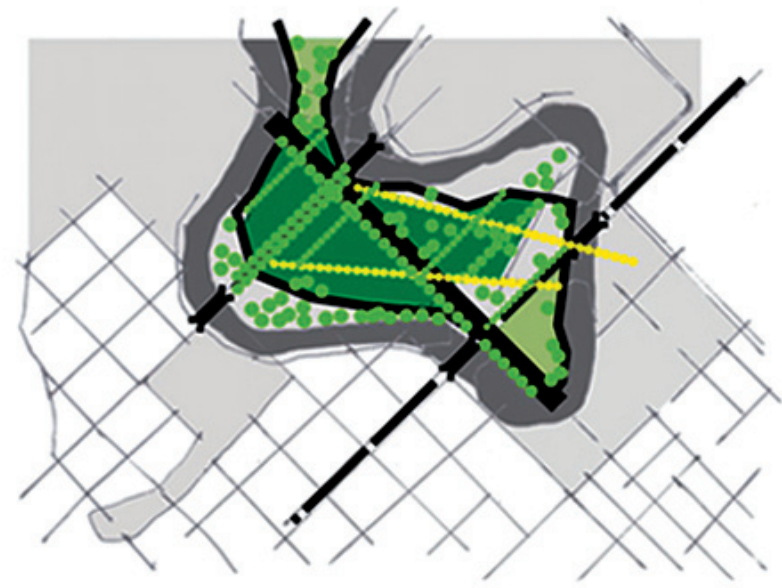

[Fines verano (especie: lluvia de oro)]

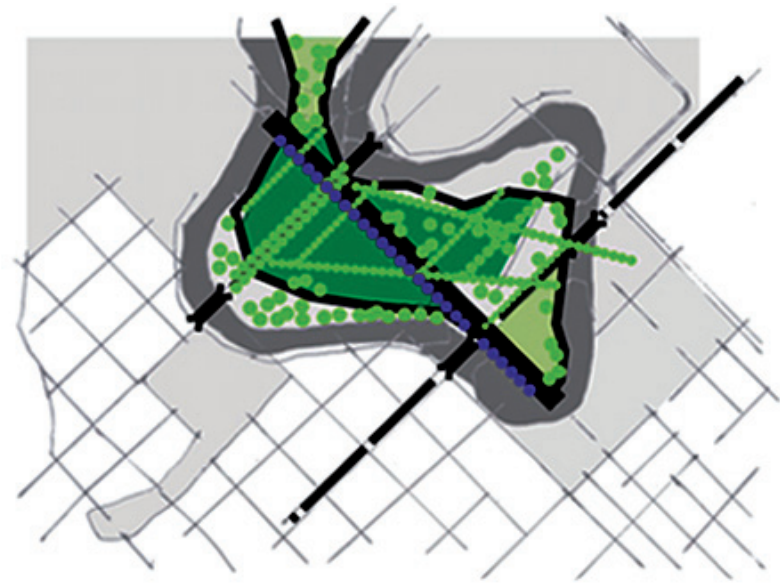

[Fines primavera (especie: jacarandá]

sin obstáculos, que forma parte de la memoria colectiva y el patrimonio natural de la ciudad.

\section{... EL EQUIPAMIENTO COMPLEMENTARIO CUMPLE DOBLE FUNCIÓN}

Sobre el eje NO-SE se ubica el equipamiento complementario que cumple una doble función, por un lado descomprime la saturación de la arteria de conexión regional, de uso muy intenso los fines de semana, y por otro detenta un rol social al tratarse de un elemento en torno del cual se ubican los espacios destinados a actividades culturales, de ocio y deportivas. Se organizó en el centro del lote dando lugar al parque de la juventud 


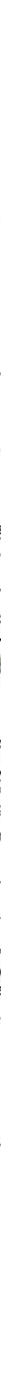

Detalle Sistema PROPUEsto
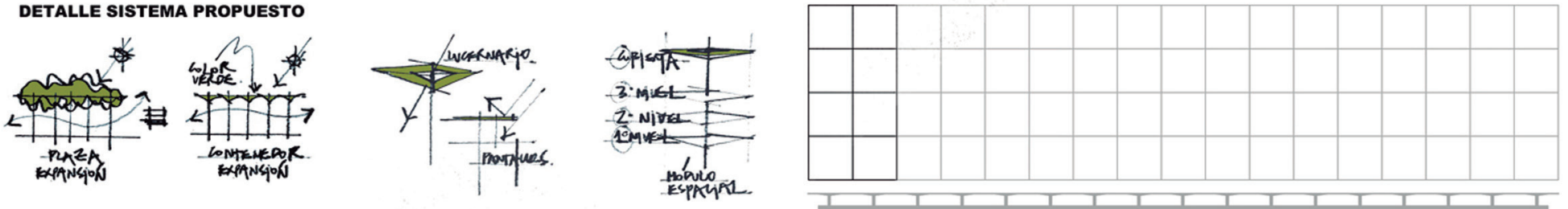

MODULOS DE SERVICIOS
FLEXIBILIDAD DE USOS: BAR, SANITARIOS PUBLICOS, INFORMES, ESTARES, MANTENIMIENTO Y SEGURIDAD.
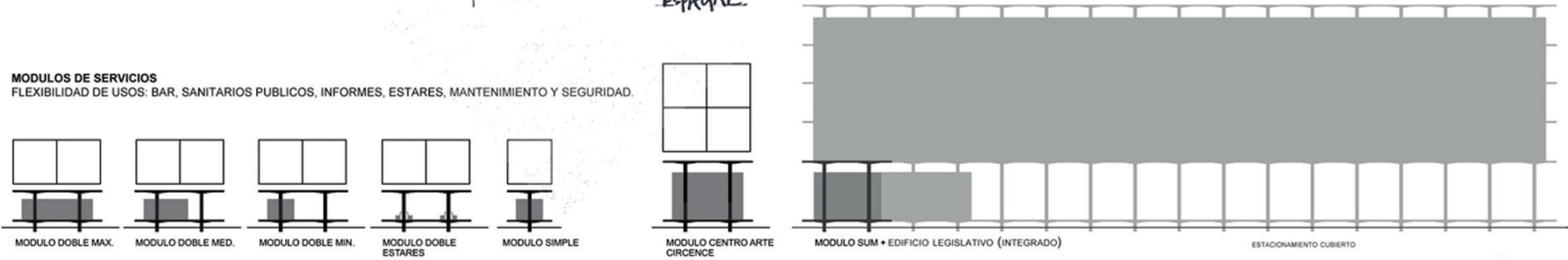

POS
OpC
ESTI
Mod
opd
EST
PAN

PARQUE URBANO DE LA DEMOCRACIA Y LA JUVENTUD PLAZA CIVICA Y POPULAR

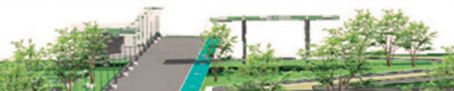

\section{(n)}





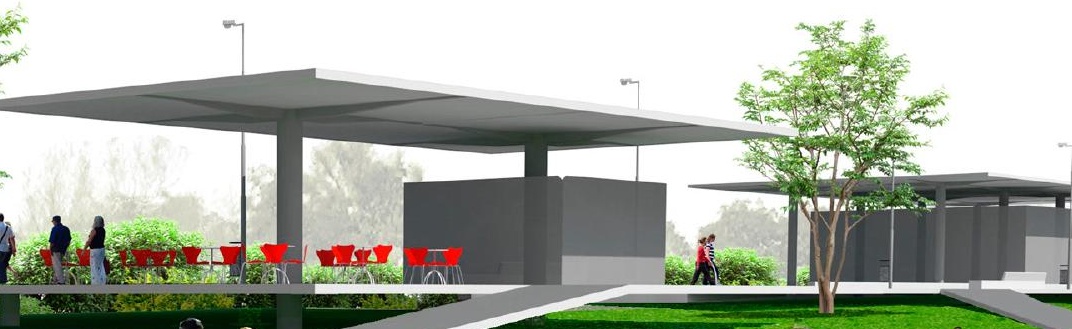

\section{CRITICA JURADO}

\section{Consideraciones generales}

El planteo refleja un profundo estudio del problema a escala urbana y del territorio.

Con respecto a la escala urbana, considera necesario "recomponer los bordes de circulación de agua -más allá de los límites del terreno -, recortados por la colonización equivocada de un territorio con ríos de llanura " y elabora estrategias de diseño urbano para su conexión con el sistema ciudad.

En la escala del lote, reproduce una troncal circulatoria lineal, para paso peatonal que reproduce el modelo de estructura urbana existente y acomete perpendicular a la Av. Sarmiento, a la que se conectan los diferentes usos, requeridos por programa.

El trabajo refleja apropiada lectura del sector urbano de emplazamiento con sus determinantes urbanos territoriales, ambientales y paisajísticos.

\section{Aspectos ambientales y paisajísticos I Adecuación al territorio natural}

La propuesta parte de un posicionamiento acertado sobre el territorio natural y propone intervenciones respetuosas de las condiciones hídricas y paisajísticas preexistentes, así como la recuperación de situaciones ambientales originales, perdidas por el impacto de la urbanización. Intenta reducir al máximo el impacto sobre las áreas más sensibles correspondientes a las cotas más bajas, introduciendo el mayor peso de las obras construidas en las cotas más altas sobre la Av. Sarmiento.

Las obras propuestas responden al mismo criterio, siendo palafíticas y livianas. Propone recuperar la continuidad del paleo cauce con el tramo que fuera fragmentado por el paso de la Av. Sarmiento.

La propuesta no inhibe la posibilidad de inundación, y de acuerdo con ella la explanada pública en cota no inundable podrá seguir siendo utilizada mientras el interior podrá ser objeto de otro tipo de usos, en coexistencia con los ciclos naturales del agua.

\section{Aspectos urbanos}

La propuesta parte de un reconocimiento integral de la localización urbana:

Considera y se posiciona correctamente sobre la Av. Sarmiento como una de las vías de acceso más im- portantes a la ciudad, pero también como uno de los espacios públicos más utilizados por la ciudadanía espontáneamente con fines deportivos y recreativos. Al respecto resuelve correctamente los accesos vehiculares y peatonales y genera una gran explanada pública que se convierte en un gran atrio o anfiteatro integrado a la avenida, reforzando y potenciando los usos espontáneos existentes y posibles.

Considera la relación del predio con su entorno inmediato, intentando resolver la integración con distintas áreas actualmente segregadas. Al respecto resulta un gesto altamente potente de integración urbana el puente propuesto en calle Liniers y la sugerencia de hacer posible algo semejante con el Barrio de la Ribera. También lo es la idea de priorizar y reforzar el cruce peatonal hacia el espacio verde público ubicado del otro lado de la Av. Sarmiento. Con ello se recupera una de las funciones primordiales de todo espacio público tratando de establecer puentes de integración frente a la fragmentación socio-espacial urbana.

- La propuesta plantea que la obra del parque sea complementaria a un proyecto de readecuación urbanística de la avenida Sarmiento con el fin de materializar de manera digna este espacio recreativo con otros sectores de la ciudad, como así también la posibilidad de prohibir en determinados días y horarios el ingreso de vehículos y motos para potenciar las prácticas saludables de ciclismo, patinaje o incluso ofrecer recorridos en bicicletas por el predio.

\section{Aspectos arquitectónicos}

La propuesta organiza la apropiación del predio de un modo simple y claro:

- mediante una cinta perimetral que propone su recorrido integralmente sin afectar las áreas más bajas y que resuelve el límite con la parcela lindera al SE - y mediante una línea recta que atraviesa todo el predio, organizando distintos espacios a partir de un planteo en espina.

La elección de estructura lineal para la conexión peatonal interna ha sido explotada con habilidad, y de manera equilibrada permite articular las actividades, empleando 
en los extremos miradores urbanos como recurso de diseño, "arranca en el agua y termina en el agua".

La explanada de referente canónico - atrio - empleada en el acceso cumple el rol de espacio de transición apropiado para descomprimir tensiones entre la avenida y el interior del lote.

El doble propósito que lleva a los proyectistas a sobreelevar el paso peatonal da cuenta del empleo de una estrategia proyectual integral.

Se resuelve el módulo "Salón de Eventos de Empleados Legislativos" a continuación de la gran explanada pública, dejando el estacionamiento próximo a la Av. Sarmiento sin producir intrusión visual al interior del predio. La propuesta permite el fácil cierre por circunstancias especiales dejando la posibilidad de uso del sector brindado a la ciudad como plaza pública.

Plásticamente, se advierte una unidad formal ponderable, que no atenta contra la riqueza del paisaje natural del sitio ni compite con ella. La cinta vehicular propuesta perimetral sobre el río y la localización de las canchas no afectan la vegetación de interés existente en el sector y los desniveles naturales; tiene un ancho mínimo de circulación en un único sentido; se materializa con un tipo de solado permeable, que promueve la marcha lenta y respetuosa de paseo.

La propuesta plantea criterios de materialización modular altamente interesantes, por su simplicidad y racionalidad. El empleo de matriz modular para la resolución de servicios aporta versatilidad de implantación y uso. 